\title{
AN ELEMENTARY METHOD FOR ESTIMATING ERROR TERMS IN ADDITIVE NUMBER THEORY ${ }^{1}$
}

\author{
ELMER K. HAYASHI
}

ABSTRACT. Let $R_{k}(n)$ denote the number of ways of representing the integers not exceeding $n$ as the sum of $k$ members of a given sequence of nonnegative integers. Using only elementary methods, we prove a general theorem from which we deduce that, for every $\epsilon>0$,

$$
R_{k}(n)-c n^{\beta} \neq o\left(n^{\beta(1-\beta)(1-1 / k) /(1-\beta+\beta / k)-\epsilon}\right)
$$

where $c$ is a positive constant and $0<\beta<1$.

Let $R_{k}(n)$ denote the number of ways of representing the integers not exceeding $n$ as the sum of $k$ members of a given sequence of nonnegative integers. Jurkat [4] has shown that $R_{k}(n)-G(n) \neq o\left(n^{\beta / 4}\right)$ whenever $k$ is an even integer, $0<\beta<2$, and $G(n)$ is a logarithmico-exponential function with $G(n) \sim c n^{\beta}, c>0$. Randol [5] has shown that $R_{k}(n)-c n^{\beta} \neq o\left(n^{\beta(1-1 / k)(1-\beta / k)}\right)$ when $m=k / \beta$ is an even integer, the given sequence of nonnegative integers is the sequence $\left\{n^{m}\right\}_{n=1}^{\infty}$, and $c$ is the volume of the $k$-dimensional solid defined by $y_{1}^{m}+y_{2}^{m}+\cdots+y_{k}^{m} \leq 1$. The corollary to our first theorem improves Jurkat's result in case $\beta<(3 k-4) /(3 k-3)$ and comes surprisingly close to Randol's result even though Randol's theorem deals only with a very special case of ours. In contrast to the methods employed by others on this type of problem (see [1]-[6]), the techniques we use here are all elementary.

We begin by defining our notation. Let $\left\{r_{1}(n)\right\}_{n=0}^{\infty}$ be a sequence of nonnegative real numbers such that if $r_{1}(n) \neq 0$, then $r_{1}(n) \geq 1$. (The lower bound 1 is chosen for convenience; any positive lower bound would suffice.) If $k$ is an integer, $k \geq 2$, define $r_{k}(n)$ by

$$
r_{k}(n)=\sum_{m_{1}+\cdots+m_{k}=n} r_{1}\left(m_{1}\right) \cdots r_{1}\left(m_{k}\right)=\sum_{m=0}^{n} r_{1}(m) r_{k-1}(n-m) .
$$

$R_{k}(n)$ will denote the summatory function of $r_{k}(n)$. Thus

$$
R_{k}(n)=\sum_{m=0}^{n} r_{k}(m)
$$

If $r_{1}(n)$ is a nonnegative integer for all $n$, we can interpret $r_{1}(n)$ as the number of occurrences of $n$ in a given sequence $\left\{a_{m}\right\}$ of nonnegative integers.

Received by the editors June 21, 1974.

AMS (MOS) subject classifications (1970). Primary $10 \mathrm{~J} 99$.

${ }^{1}$ This paper is, with minor changes, part of the author's Ph.D. dissertation, written at the University of Illinois, Urbana, under the direction of Professor Paul T. Bateman. 
In this case, $r_{k}(n)$ denotes the number of ways $n$ can be represented as the sum of $k$ elements of the sequence $\left\{a_{m}\right\}$. It is interesting to note, however, that the proofs of our theorems do not require $r_{1}(n)$ to be integer-valued.

Finally, define $\Delta G(n)=G(n)-G(n-1)$ and $\Delta^{2} G(n)=\Delta(\Delta G(n))$. We write $f(n) \ll g(n)$ when $f(n)$ is less than a positive multiple of $g(n)$ for all sufficiently large $n$. Our main result is

Theorem 1. Let $0<\beta \leq 1-\delta<1$. If $R_{k}(n)=G(n)+v(n)$, with $v(n)=$ $o(G(n))$, if $n^{\beta} \ll G(n) \ll n^{1-\delta}$, and if $\Delta^{2} G(n) \leq 0$ for all sufficiently large $n$, then for every $\epsilon>0$ we have

$$
v(n) \neq o\left(n^{\delta \beta(1-1 / k) /(1-\beta+\beta / k)-\epsilon}\right) .
$$

Proof. By hypothesis, there exists $n_{0}>0$ such that $\Delta^{2} G(n) \leq 0$ for $n \geq$ $n_{0}$, i.e., $\Delta G(n)$ is nonincreasing for $n \geq n_{0}$. Thus for $n \geq n_{0}$ we have

$$
\left(n-n_{0}\right) \Delta G(n) \leq \sum_{m=n_{0}+1}^{n} \Delta G(m)=G(n)-G\left(n_{0}\right),
$$

and hence

$$
\Delta G(n) \ll G(n) / n .
$$

Choose $x \in Z^{+}$such that $r_{1}(x+1) \neq 0$ and assume $2<y<x$, where $y$ is an integer to be specified later. Since $R_{k}(n) \sim G(n)$ and $G(n) \rightarrow \infty$ as $n \rightarrow \infty$, there exist arbitrarily large $n$ for which $r_{1}(n) \neq 0$. Hence $x$ can be taken arbitrarily large. Using (2) and (1), we see that

$$
\begin{aligned}
R_{k}(x+y)-R_{k}(x) & =\sum_{m=x+1}^{x+y} r_{k}(m)=\sum_{m=x+1}^{x+y} \sum_{j=0}^{m} r_{1}(j) r_{k-1}(m-j) \\
& \geq r_{1}(x+1) \sum_{m=0}^{y-1} r_{k-1}(m)=r_{1}(x+1) R_{k-1}(y-1) .
\end{aligned}
$$

At first glance, the above estimate might seem to be rather crude. However, since by hypothesis $G(n)=o(n)$, and hence $R_{k}(n)=o(n)$, it follows that it is very unlikely that $r_{1}(n) \neq 0$ for very many $n$ between $x$ and $2 x$. Thus the above estimate is good when $R_{k}(n)$ is significantly smaller than $n$ in magnitude.

For the moment, let us assume that we know

$$
R_{k-1}(y) \gg\left(R_{k}(y)\right)^{1-1 / k} \text {. }
$$

Now using $G(n) \gg n^{\beta}$ and recalling $R_{k}(n) \sim G(n)$, we obtain, for sufficiently large $y$,

$$
R_{k}(x+y)-R_{k}(x)>\left(R_{k}(y-1)\right)^{1-1 / k} \gg(G(y-1))^{1-1 / k} \gg y^{\beta(1-1 / k)} .
$$

On the other hand, if $v(n)=o\left(n^{\alpha}\right)$, then using the fact that $\Delta G(n)$ is nonin- 
creasing and applying (4), we get

$$
\begin{aligned}
R_{k}(x+y)-R_{k}(x) & =G(x+y)-G(x)+v(x+y)-v(x) \\
& \leq y \Delta G(x)+o\left(x^{\alpha}\right)<y G(x) / x+o\left(x^{\alpha}\right) .
\end{aligned}
$$

Thus we have, for sufficiently large $y$,

$$
y^{\beta(1-1 / k)}<y G(x) / x+o\left(x^{\alpha}\right) .
$$

We now choose $y$ so that

$$
y G(x) / x=o\left(y^{\beta(1-1 / k)}\right),
$$

say

$$
y=\left[(x / G(x))^{1 /(1-\beta+\beta / k)-\epsilon}\right], \quad \epsilon>0 .
$$

It is easily verified that $y<x$, and when $\epsilon$ is small, $y$ grows large with $x$. With our choice of $y$, we see that a contradiction of (6) will occur if $\alpha<$ $\delta \beta(1-1 / k) /(1-\beta+\beta / k)$, since then for sufficiently small $\epsilon$ we have

$$
\begin{aligned}
x^{a} & \leq x^{\delta \beta(1-1 / k)(1 /(1-\beta+\beta / k)-\epsilon)} \\
& <(x / G(x))^{\beta(1-1 / k)(1 /(1-\beta+\beta / k)-\epsilon)}<y^{\beta(1-1 / k)} .
\end{aligned}
$$

The proof of the theorem will be complete if we can verify (5). In fact we shall show

$$
\left(R_{k}(y)\right)^{k-1} \leq(k-1)\left(R_{k-1}(y)\right)^{k} .
$$

We write

$$
\left(R_{k}(y)\right)^{k-1}=\left(\sum_{a_{1}+\cdots+a_{k^{-}} y} r_{1}\left(a_{1}\right) \cdots r_{1}\left(a_{k}\right)\right)^{k-1}
$$

If we multiply out the right side of the last equation, we see that a typical term of $\left(R_{k}(y)\right)^{k-1}$ is $\Pi_{i=1}^{k-1}\left\{\Pi_{j=1}^{k} r_{1}\left(a_{i j}\right)\right\}$, where $\Sigma_{j=1}^{k} a_{i j} \leq y$ for $i=1,2$, $\cdots, k-1$. Now

$$
\sum_{i=1}^{k-1} \sum_{j=1}^{k} a_{i j} \leq \sum_{i=1}^{k-1} y=(k-1) y .
$$

It follows that for some $t \leq k-1$ we have $\sum_{i=1}^{k-1} a_{i t} \leq y$, and for $i=1, \cdots$, $k-1$, we clearly have $\Sigma_{j=1 ; j \neq t}^{k} a_{i j} \leq \sum_{j=1}^{k} a_{i j} \leq y$. Thus

$$
\left\{\prod_{m=1}^{k-1} r_{1}\left(a_{m t}\right)\right\} \prod_{i=1}^{k-1}\left\{\prod_{j=1 ; j \neq t}^{k} r_{1}\left(a_{i j}\right)\right\}
$$

is a term of $\left(R_{k-1}(y)\right)^{k}$. Hence each term of $\left(R_{k}(y)\right)^{k-1}$ occurs as a term of $\left(R_{k-1}(y)\right)^{k}$. However, since $t$ could have any of $k-1$ different values, it follows that we may have associated as many as, but no more than, $k-1$ different terms of $\left(R_{k}(y)\right)^{k-1}$ with the same term of $\left(R_{k-1}(y)\right)^{k}$. Therefore we have 


$$
\left(R_{k}(y)\right)^{k-1} \leq(k-1)\left(R_{k-1}(y)\right)^{k} .
$$

This completes the proof of Theorem 1 .

The constant $k-1$ in (8) is probably not best possible. The correct constant is most likely 1 , but we have only been able to prove this in certain special cases. For example, if $r_{1}(n)=1$ for all $n$, then

$$
\begin{aligned}
\left(R_{k}(n)\right)^{k-1} & =((n+1)(n+2) \cdots(n+k) / k !)^{k-1} \\
& =(1+n / 1)^{k-1}(1+n / 2)^{k-1} \cdots(1+n / k)^{k-1} \\
& \leq(1+n / 1)^{k}(1+n / 2)^{k} \cdots(1+n /(k-1))^{k} \\
& =((n+1)(n+2) \cdots(n+k-1) /(k-1) !)^{k} \\
& =\left(R_{k-1}(n)\right)^{k} .
\end{aligned}
$$

Taking $\delta=1-\beta$ in Theorem 1, we obtain the following

Corollary. Let $0<\beta<1$. If $R_{k}(n)=G(n)+v(n)$ with $v(n)=o(G(n))$, if $G(n) \sim c n^{\beta}$ with $c>0$, and if $\Delta^{2} G(n) \leq 0$ for all sufficiently large $n$, then for every $\epsilon>0$,

$$
v(n) \neq o\left(n^{\beta(1-\beta)(1-1 / k) /(1-\beta+\beta / k)-\epsilon}\right) .
$$

In our second theorem, we prove that even if very little is known about the exact order of magnitude of $G(n)$, we can still claim that $v(n) \neq O(1)$.

Theorem 2. If $R_{k}(n)=G(n)+v(n)$ with $v(n)=o(G(n))$, if $G(n)=o(n)$, but $G(n) \rightarrow \infty$ as $n \rightarrow \infty$, and if $\Delta^{2} G(n) \leq 0$ for all sufficiently large $n$, then $v(n) \neq O(1)$.

Proof. Suppose there exists $M>0$ such that $|v(n)| \leq M$ for all $n$. Recall that in the proof of Theorem 1 , we showed that $\Delta G(n)<<G(n) / n$ is a consequence of the hypothesis $\Delta^{2} G(n) \leq 0$ for all sufficiently large $n$. Since we also have $G(n)=o(n)$, we conclude $\Delta G(n)=o(1)$. The hypotheses $R_{k}(n) \sim$ $G(n)$ and $G(n) \rightarrow \infty$ as $n \rightarrow \infty$ guarantee that we have $r_{1}(n) \neq 0$, and hence $r_{1}(n) \geq 1$, for infinitely many $n$. Thus there exist integers $a$ and $A$ such that $R_{1}(A)-R_{1}(a) \geq 2 M+1$. Choose $N$ so that $r_{1}(N) \neq 0$. Since $R_{1}(N) \geq 1$, we have

$$
2 M+1 \leq R_{1}(A)-R_{1}(a)=\sum_{a<n \leq A} r_{1}(n) \leq \sum_{a<n \leq A} r_{1}(n)\left(r_{1}(N)\right)^{k-1} .
$$

Using (1) and (2), we see that every term of the last sum is a term of $R_{k}((k-1) N+A)-R_{k}((k-1) N+a)$. Since $N$ can be chosen arbitrarily large, we therefore have 


$$
\begin{aligned}
2 M+1 & \leq R_{k}((k-1) N+A)-R_{k}((k-1) N+a) \\
& =G((k-1) N+A)-G((k-1) N+a)+v((k-1) N+A)-v((k-1) N+a) \\
& \leq(A-a) \Delta G((k-1) N+a)+2 M \\
& =o(1)+2 M \text { as } N \rightarrow \infty .
\end{aligned}
$$

The assumption $v(n)=O(1)$ has led us to a contradiction. Therefore we conclude $v(n) \neq O(1)$.

The author expresses his great appreciation to Dr. Paul T. Bateman for his encouragement and many helpful suggestions during the preparation of this paper.

\section{REFERENCES}

1. P. T. Bateman, The Erdös-Fuchs theorem on the square of a power series (to appear).

2. P. T. Bateman, E. E. Kohlbecker and J. P. Tull, On a theorem of Erdös and Fuchs in additive number theory, Proc. Amer. Math. Soc. 14 (1963), 278-284. MR 26 \#2417.

3. P. Erdös and W. H. J. Fuchs, On a problem of additive number theory, J. London Math. Soc. 31 (1956), 67-73. MR 17, 586.

4. W. Jurkat, (to appear).

5. B. Randol, A lattice-point problem. II, Trans. Amer. Math. Soc. 125 (1966), 101-113. MR $34 \# 1292$.

6. R. C. Vaughan, On the addition of sequences of integers, J. Number Theory 4 (1972), 1-16. MR 44 \#5291.

DEPARTMENT OF MATHEMATICS, WAKE FOREST UNIVERSITY, WINSTON-SALEM, NORTH CAROLINA 27109 